\title{
Uso de las tic en el estudio del derecho penal
}

\author{
Abigail Gaytán Martínez, Jorge Alberto Pérez Pinto, \\ Iván Noé Martínez Ponce y Francisco Javier Itzamna Caamal Torre
}

\section{Resumen}

La educación en el mundo actual requiere de sistemas teóricos y tecnológicos que permitan una acción comunicativa eficiente. En este tenor de ideas, las tecnologías se presentan como herramienta con alto valor de información y de comunicación. Así, éstas y, en particular, su empleo reflexivo, se convierten en valores de uso y cambio que permiten al proceso de enseñanza-aprendizaje convertirse en un proceso dinámico y autorregulable. En este artículo se presenta una experiencia del uso de las tecnologías de la información y la comunicación (TIC) para la enseñanza del derecho penal sustantivo y adjetivo.

Palabras clave: TIC, derecho penal sustantivo, derecho penal adjetivo, vinculación social.

\section{ITC USAGE IN THE STUDY OF CRIMINAL LAW}

\begin{abstract}
Education in today's world requires theoretical and technological systems that allow an efficient communicative action. In this tenor of ideas, technologies are presented as a tool with a high value for information and communication. Thus, technologies, in particular their reflective use, become values of use and change that allow the teaching-learning process to become a dynamic and self-regulating process. This article presents an experience of the use of information and communication technologies (ITC) for the teaching of substantive and adjective criminal law.
\end{abstract}

Keywords: ITC, substantive criminal law, adjective criminal law, social linkage.

DOI: http://doi.org/10.22201/codeic.16076079e.2019.v20n3.a9 
Abigail Gaytán Martínez

abigailgaytan@gmail.com

Docente Investigador de Tiempo Completo, Unidad Académica de Derecho, Universidad Autónoma de Zacatecas "Francisco García Salinas".

Jorge Alberto Pérez Pinto

pinzac57@gmail.com

Docente Investigador de Tiempo Completo, Unidad Académica de Derecho, Universidad Autónoma de Zacatecas "Francisco García Salinas".

\section{Iván Noé Martínez Ponce}

maquio1975@hotmail.com

Docente Investigador de Tiempo Completo, Unidad Académica de Derecho, Universidad Autónoma de Zacatecas "Francisco García Salinas".

\section{Francisco Javier Itzamna Caamal Torres}

Ifcaamalt@hotmail.com

Docente Investigador de Tiempo Completo, Unidad Académica de Derecho, Universidad Autónoma de Zacatecas "Francisco García Salinas".

\section{Introducción}

La educación universitaria debe formar con base en competencias con el objetivo de fomentar el aprendizaje permanente a lo largo de la vida, fundamentándose en una educación centrada en la construcción e integración de diversos recursos: capacidades, habilidades, actitudes, etcétera, más que en la acumulación de conocimientos (Leyva, 2015).

En particular, los estudiantes de Derecho deben tener una experiencia educativa en el aula que les permita vincularse con la realidad social de su mundo profesional. Así, podemos afirmar que en las universidades hemos de impulsar procesos educativos que tengan como punto de partida la formación, con base en las competencias necesarias y pertinentes, de espacios educativos para el desempeño profesional de los alumnos y docentes, más allá de regiones y naciones.

La Asociación Nacional de Universidades e Instituciones de Educación Superior (ANUIES) propuso la adecuación de la educación superior para que esté vinculada con el mundo y sus problemas; así como con el desarrollo social, económico y cultural del país; con problemas y necesidades de las regiones; y con el desarrollo de redes de vinculación con otras instituciones de educación 
"Uso de las tic en el estudio del derecho penal"

Abigail Gaytán Martínez, Jorge Alberto Pérez Pinto,

superior nacionales y extranjeras. En suma, una educación superior del mundo global al servicio del desarrollo de México y de sus regiones (ANUIES, 2012).

Es por ello que las instituciones de educación superior tienen la obligación de aparejar los modelos educativos a esa visión, para responder a los retos que presenta la sociedad mexicana en un entorno de globalización del conocimiento (ANUIES, 2012).

Las instituciones de educación superior tienen, entonces, la imperiosa necesidad de reelaborar los objetivos, misiones, visiones y estrategias, de sus planes de estudio, con la finalidad de educar con base en procesos educativos: I) comparables a nivel internacional; ॥) con base en habilidades y destrezas medibles y demostrables; ॥I) con transparencia en el manejo del capital humano y material; Iv) construidos conforme a los requerimientos de la sociedad; v) en torno a una visión clara de vinculación y extensión; y vi) bajo convergencias interinstitucionales, de acuerdo con ANUIES (2012) y como lo señaló el doctor Alejandro Aguilera Galaviz, Secretario Académico de la Universidad Autónoma de Zacatecas (Malinali, 2016). Así, y sólo así, podremos hacer de la academia el lugar donde el conocimiento permita la elaboración de mejores experiencias laborales y de vida.

En efecto, el aula y las herramientas tecnológicas permiten una vinculación permanente y sólida entre el alumno y las experiencias de aprendizaje. Las TIC, más allá de constituirse en la operación de tecnologías o herramientas tecnológicas, deben de permitir una comunicación y vinculación constante entre el docente, alumno y la experiencia de aprendizaje. Con ello las herramientas tecnológicas se integran al proceso educativo y facilitan: a) hacer del proceso educativo una experiencia permanente; b) dar soporte al alumno y al docente, para la vinculación permanente respecto a las temáticas del programa; c) ampliar el proceso reflexivo, y con ello impulsar el desarrollo de competencias como uso de tecnologías, reflexión y capacidad de lectura-redacción; d) conformar un espacio crítico que haga análisis a profundidad de temas frontera; y e) vincular la experiencia de aprendizaje con la realidad social.

\section{El precedente}

Transcurre el año 2016 y por enésima ocasión nos reunimos cuatro compañeros docentes para comentar, armados de sendas tazas de café-claro-, los problemas cada vez más agudos que evidencia la Universidad Autónoma de Zacatecas y nuestra entidad de adscripción, la Unidad Académica de Derecho.

Se toma una decisión: basta de quejas, de análisis objetivos -o no- de lo que ocurre por la falta de financiamiento a la institución o por la ausencia de apoyos para el desarrollo correcto de academia e investigación en Derecho. Ahora sólo se aceptarían propuestas para buscar mejorar en lo institucional, en lo individual y en lo colectivo; para innovar; y para apoyar a maestros y estudiantes. 
"Uso de las tic en el estudio del derecho penal" Abigail Gaytán Martínez, Jorge Alberto Pérez Pinto,

Se pasa entonces de pláticas rebosantes de temores, quejas y politiquerías a otras plenas de identificación de problemas, ausencias y rezagos, acompañadas con propuestas de solución. Entre otros, se identifican los inconvenientes, motivos del presente esfuerzo y sus posibles soluciones, lo cuales se enumeran a continuación:

1. La impartición de las materias seriadas del área de Derecho Penal sufre una interrupción cronológica.

a) Los alumnos, al iniciar el curso de Derecho Penal ॥, Delitos en particular, argumentan o no recordar las enseñanzas recibidas en su curso general de Derecho Penal, o manifiestan que el maestro no fue capaz de transmitirles el conocimiento.

b) Al iniciar el curso de Derecho Procesal Penal repetían la historia, pero ahora incluyendo a los maestros de su asignatura de Delitos en particular.

c) Este hecho nos lleva al inconveniente del retraso en la impartición de las asignaturas de Derecho Penal ॥ y de Derecho Procesal Penal, ya que los docentes debían comenzar con un repaso de la materia anterior y al término comenzar con la materia propia.

Ahora bien, ¿eran ciertos los comentarios de los alumnos? Los programas, resultan ser los mismos y se imparten en los tiempos señalados en el calendario escolar; al practicar exámenes colegiados, se constata que los maestros del área imparten conocimientos apegados a los programas, lo que permitió concluir que las excusas no son válidas. Entonces, ¿dónde radica el problema?

La respuesta la encontramos en un ilógico plan de estudios, que establece una pausa de un semestre en la continuidad de las materias seriadas que comentamos. Este hecho naturalmente impide que los alumnos cuenten con los conocimientos frescos de los temas abordados en la materia anterior.

La solución se advertía fácil: diseñar un plan de estudios que eliminara la interrupción. Se planteó, pero nos informaron que sería en 2017 cuando se convocaría al Congreso de Reforma, ámbito universitario adecuado para un cambio en el plan de estudios y donde se podría gestar una solución de la problemática.

2. La existencia de una enseñanza tradicional del Derecho, practicada a través de la clase magistral, donde el docente tiene el carácter expositivo y dominante de la actividad, siendo pocos los profesores que han adoptado herramientas tecnológicas. Aún en esos pocos casos, la herramienta es utilizada para reproducir el modelo tradicional (González y Castillo, 2017).

3. La poca capacidad en los estudiantes para relacionar lo que ocurre en un contexto social globalizado, con el diseño de políticas y su impacto en el contenido o formulación de normas.

Debemos tener claro que la educación en el siglo xxl nos compele a impulsar procesos educativos estandarizados que hagan de los egresados de 
"Uso de las tic en el estudio del derecho penal" Abigail Gaytán Martínez, Jorge Alberto Pérez Pinto,

sus distintos niveles hombres y mujeres capacitados para desenvolverse en múltiples escenarios sociales y trabajar en cualquier ambiente laboral.

4. La insuficiente vinculación de la Unidad Académica de Derecho con la sociedad. Es claro que en la era de la tecnología, y en un esquema neoliberal de la construcción de las políticas públicas, se ha dejado de lado la participación activa de las universidades, escuelas y facultades, debido a que éstas no han sabido comunicar las producciones científicas, técnicas o artísticas que en sus espacios se formulan.

Aunado a lo anterior, se debe reconocer que los requerimientos del mercado apuestan más por la inmediatez que por la construcción crítica y reflexiva de productos que atiendan a las necesidades de la sociedad.

5. La casi nula capacidad de nuestros alumnos para resolver casos prácticos. Existe un reclamo extendido, y con causa justificada, de que en las aulas no se prepara a los alumnos para enfrentar los problemas reales. Esto, más que un problema de enseñanza, es resultado de un rompimiento comunicativo entre la teoría y la práctica.

No se piense que se pretende lograr una comunicación con la totalidad de la sociedad. Sí, en cambio, llegar a las personas que encuentren en las producciones académicas elementos importantes para la resolución de problemáticas en su vida cotidiana, como lo serían los problemas legales.

Apremia, entonces, reconocer que las necesidades de información son distintas y deben ser atendidas por los profesionales mediante el uso de los varios medios de comunicación y transmisión de conocimiento existentes.

La respuesta a las cinco problemáticas identificadas, coincidimos, estriba en el uso de tecnologías de la información y la comunicación (TIC).

\section{La implementación}

En este marco fue sencillo coincidir en la solución, pero es muy diferente llevarla a la práctica. Ante la multiplicidad de sistemas de comunicación, fue necesario conocer y escoger la herramienta que permitiera la comunicación entre los coautores y los posibles usuarios.

Desconocíamos los pormenores de la utilización de las Tic en la educación, al grado de que el coautor que propuso la solución se autodefinió como "un analfabeta funcional de la informática". Debimos investigar, aprender y resolver nuevos retos, y a la par entender y aceptar que la implementación requería dedicar tiempo adicional al destinado a las labores ordinarias.

Se superó el primer desafío que según el informe Horizon para Latinoamérica debe contemplarse para la incorporación de las TIC en las instituciones educativas: la formación docente en el uso de medios digitales en los procesos de enseñanza y aprendizaje (García et al., 2010), la cual consiste en: 


\section{Transformación de roles}

a) El docente debe utilizar de forma eficiente las Tic en su práctica educativa y proponer la construcción intencional de su uso (UNESCO, 2016). Para el caso, la finalidad de la herramienta consiste en ayudar al alumno a resolver el problema planteado e iniciarlo en la comprensión e inserción de lo que es una sociedad del conocimiento, aquella "que se nutre de sus diversidades y capacidades [...] comprende dimensiones sociales, éticas y políticas" (UNESCO, 2005).

b) El educando debe participar, exigiendo que quede atrás la enseñanza tradicional del Derecho y transformándose en un sujeto activo de su propio aprendizaje para incrementar notablemente sus conocimientos y desarrollar agudeza crítica que le permita elaborar nuevas soluciones a los problemas que surgen en la cambiante vida social de nuestra época (Saavedra, 2016).

2. Así pues, se crea una herramienta tecnológica básica, un blog cuyos contenidos, breves y con ejemplos prácticos, se dirigieron a refrescar conocimientos para ayudar a superar la pausa entre semestres y a iniciar al educando en la aplicación práctica de la teoría del delito, con la clasificación de tipos penales.

\section{Análisis inicial de los datos}

La herramienta, UAZ, DERECHO PENAL, con dirección http://uazderechopenal. blogspot.mx, inició el 3 de agosto de 2016 y, en su primer mes de vida, arrojó los datos que se muestran en la figura 1.

Figura 1. Análisis inicial de los datos.
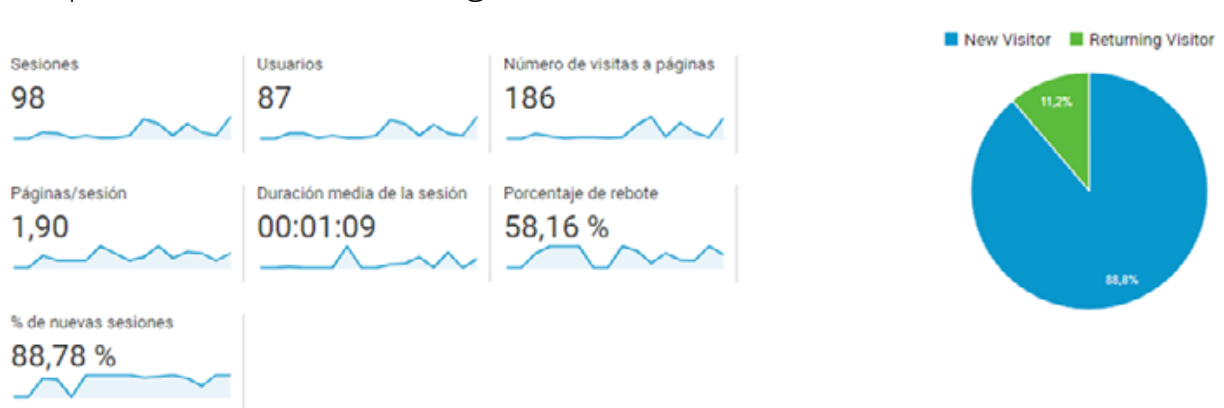

Hubo un buen avance para el poco tiempo de inicio del blog, pero no era suficiente, ya que el reto implicaba llegar al mayor número posible de los 2097 alumnos que cursaban los semestres del cuarto al décimo de la Licenciatura en Derecho.

Para corregir rumbo, identificamos que el sistema operativo a través del cual accedían los usuarios era principalmente Android. Al aplicar una encuesta a 120 alumnos de la Unidad Académica, los resultados indicaron que el usuario prefiere el uso de redes sociales. Incorporamos entonces la herramienta a Facebook, con el usuario DERECHO PENAL ABIGAIL, y comenzó el envío de solicitudes de amistad. 
Figura 2. Datos 2016

y 2019 de usuarios, sesiones, visitas y retornos.
Figura 3. Muestra la ubicación geográfica 2016 y 2019 de usuarios, un crecimiento de 14 a 61 países.
Se enlazó el blog a Facebook, consultable a la fecha aquí. El cambio funcionó, las solicitudes de amistad fueron aceptadas por alumnos y exalumnos integrados a despachos jurídicos locales.

\section{Los avances cuantitativos}

- El blog. A tres meses de su inicio se registraron 997 usuarios, con 1623 sesiones y 3560 visitas; para beneplácito de los autores, alumnos y exalumnos compusieron $91 \%$ de los usuarios y el alcance se extendió a otros estados y a 14 países, como se puede ver en las figuras 2 y 3.
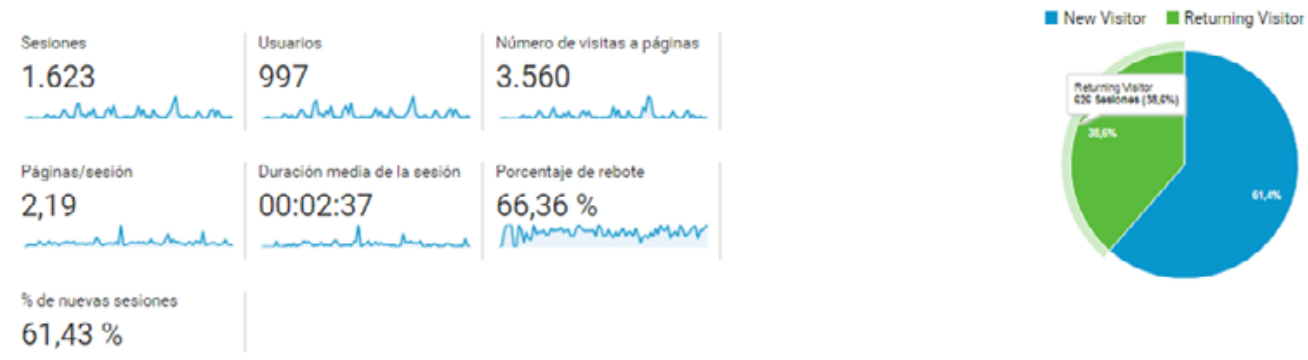

\section{$61,43 \%$}
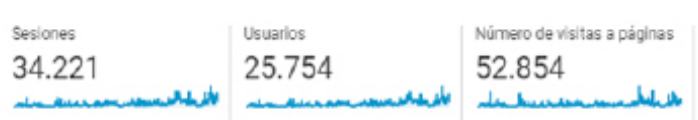

Duración media de la sesión 00:01:28 Porcentaje de rebote

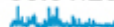
$81,22 \%$ sce nuevas sesiones $75,26 \%$
Páginas/sesiòn 1,54

- New Visitor a Retuming Visitor
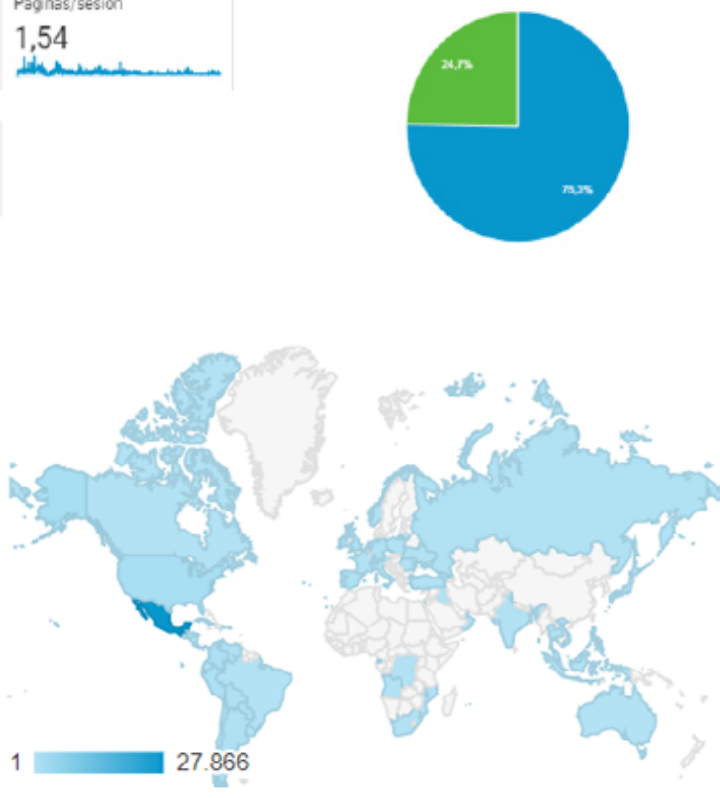

Los usuarios de la herramienta en México se ubican en 18 estados, pero el grueso de ellos, 638, son vecinos del Estado de Zacatecas; además, los resultados de una encuesta realizada a través de Facebook, indicaron que ya se cubría 28.3\% de alumnos inscritos en nuestra Unidad Académica del cuarto al décimo semestre. Un universo de 593 alumnos quedó cubierto en tres meses. 
Figura 4. Muestra la ubicación 2016 y 2019 de los usuarios en la República Mexicana.
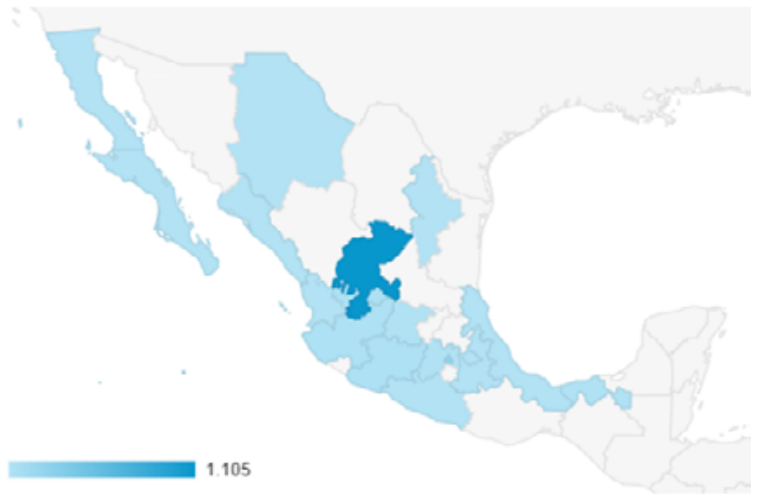

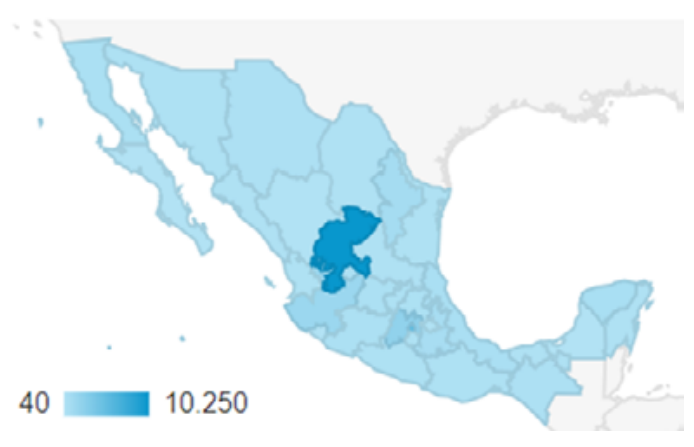

- La herramienta en el perfil de Facebook. A octubre de 2016 sumaban 587 seguidores con un alcance orgánico de 5 433. A marzo de 2019, sumaban 2 453 seguidores, como se muestra en la figura 5.

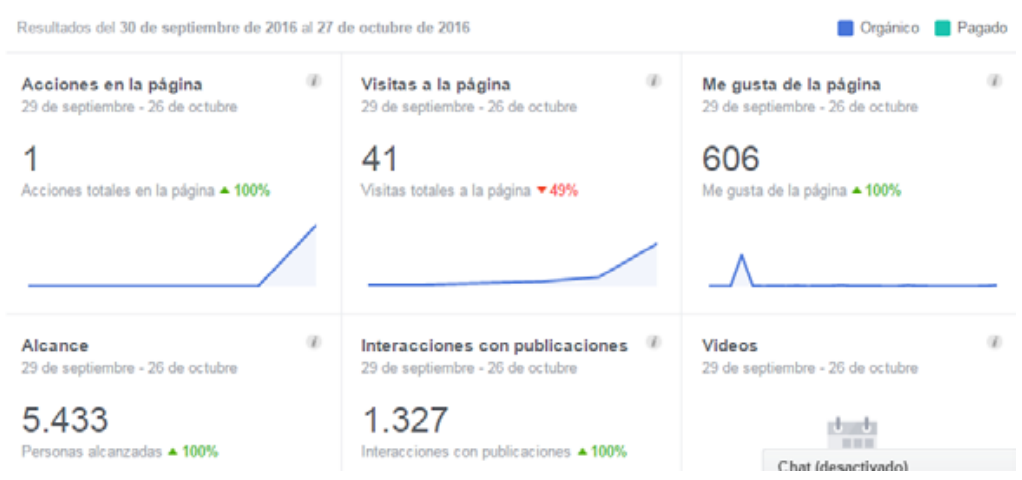

Total de seguidores de la página hasta hoy: 2,453

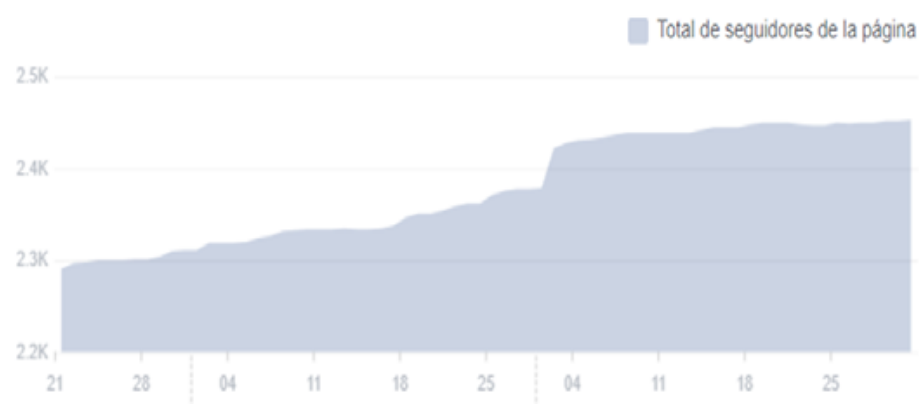

515 fans en promedio se conectaban diariamente hacia octubre de 2016. Para 2019 el número incrementó, como se puede observar en la figura 6. 
Figura 6. Medición semanal que ingresa al perfil público 2016 y 2019.

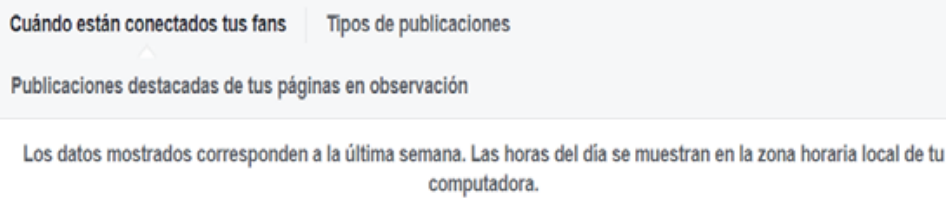

DiAS

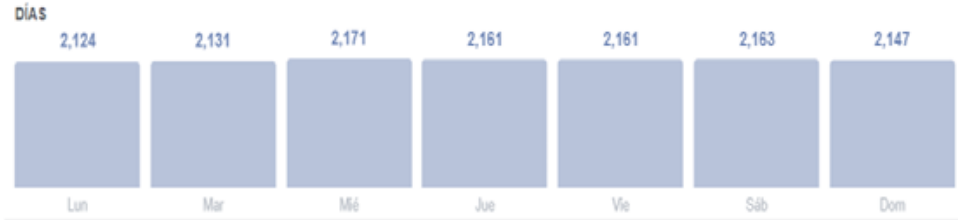

Cuando estan eoneotados tus fans Tipos de publicaciones

Publicaciones destacadas de tus páginas en observación

Los datos mostrados corresponden a la última semana. Las hora del dia se muestran en la zona horaria local de tu computadora. DiAs

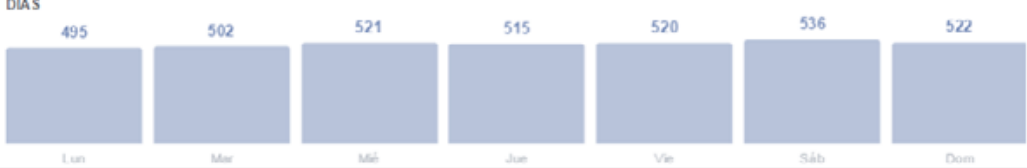

\section{Las publicaciones}

Para marzo de 2019 los autores habían publicado 112 artículos con temas de análisis orientados a la finalidad de la herramienta y con particular énfasis en la teoría del delito. Las publicaciones se relacionan con la norma penal zacatecana vigente, lo cual está en consonancia con que la mayoría de los usuarios son residentes de Zacatecas. A la par, se transitó hacia la comunicación multimedia mediante la elaboración y publicación de videos.

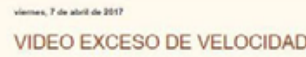

Estimados amigos del blog, hoy, a la inversa y por ser un archivo que por su extension no puede subirse a traves del blog UAZ, DERECHO PENAL, estamos compartiendo información sobre la conduection a exeeso de velocidar considerado en zacenecas como un decilo, te pedimos que puedas

nttps://www. facebook.com/abigall. perezpinto.ivannoe.caamall

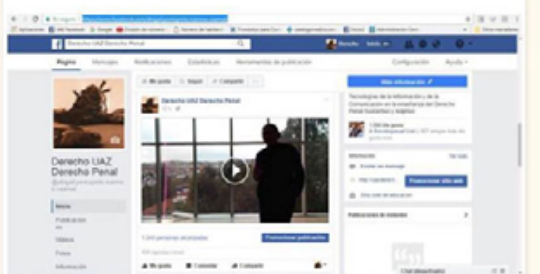

LO DEJAMOS PARA TU REFLEXION Y COMENTARIOS
Figura 7. Ejemplo de publicaciones que integran el blog. 


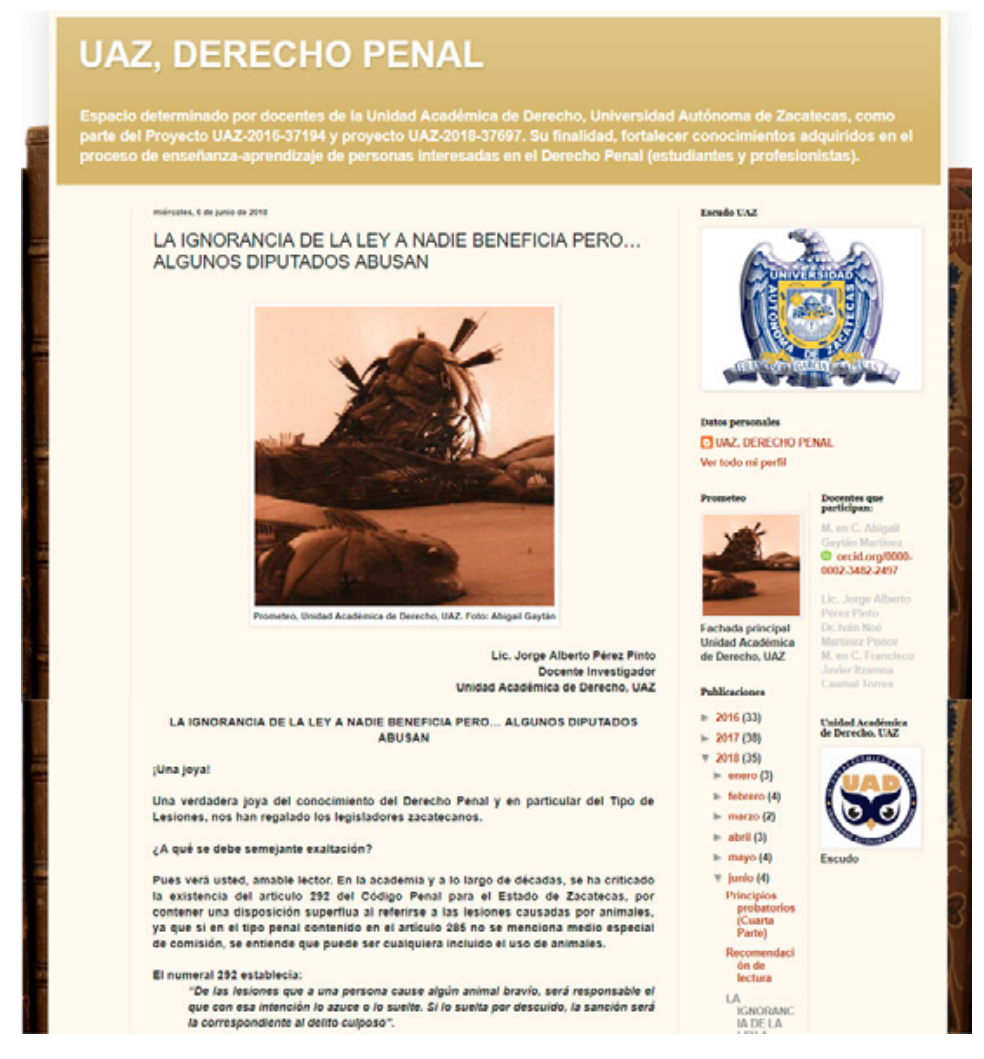

\section{Los resultados y experiencias}

Los números mostrados son satisfactorios, la publicación interesó a miles en una amplia geografía, pero por sí solos no muestran si los fines se han cumplido o se encuentran en vía de hacerlo. A continuación, nos referiremos a los logros alcanzados con nuestra propuesta, así como algunas perspectivas.

Logro 1. Seis semestres de la Licenciatura en Derecho han transcurrido desde la aparición del blog y, en las aulas, presenciamos el incremento, en cada ciclo, del número de alumnos que lo consultan.

Sin embargo, aún quedan algunos pendientes: no se ha emitido la convocatoria para realizar el foro de reforma, anunciado para 2017, por lo que continúa la interrupción de un semestre en el estudio de las materias de Derecho Penal. El servicio de internet no llega a todas las aulas, lo que limita la posibilidad de consulta en clase y, además, de manera deplorable, algunos maestros prohíben el uso de las Tic durante el desarrollo de su cátedra.

Logro 2. Los alumnos que en el presente semestre acudieron a audiencias de juicio oral por cursar la asignatura de Derecho Procesal Penal tuvieron a su disposición la colaboración "Interrogatorio y contrainterrogatorio". Este artículo se reprodujo en 1100 ocasiones con 1497 personas alcanzadas, lo que da indicios de la necesidad de incluir más artículos sobre el tema. 
"Uso de las tic en el estudio del derecho penal" Abigail Gaytán Martínez, Jorge Alberto Pérez Pinto,

Logro 3. Los artículos en formato audiovisual tuvieron mayor impacto que los puramente textuales. Baste para ejemplificar que el video relativo a los delitos de tránsito alcanzó 6500 reproducciones y su texto sólo 1430.

No obstante, hace falta convencer a nuestras autoridades de la conveniencia de proporcionar los recursos humanos y materiales necesarios para continuar con el desarrollo del material multimedia.

Logro 4. Se alcanzó la internacionalización de la institución y ahora se debe transitar del conocimiento del número de usuarios de otros países a la identificación de lo que sus lecturas en el blog les significan a los lectores, así como motivar la interacción.

Logro 5. Los alumnos opinaban sobre artículos escritos para su reflexión en Google+ y en Facebook y, a la fecha, se optó por desaparecer la primera herramienta. Una alumna de la maestría en Ciencia Jurídico-Penal decidió tomar el artículo "¿El delito de parricidio debe desaparecer de nuestro catálogo de delitos?" como base para la elaboración de su tesis de grado.

Logro 6. El artículo "Dos motivos de anticonstitucionalidad del nuevo Reglamento General de la Ley de Transporte, Tránsito y Vialidad del Estado de Zacatecas", publicado el 30 de noviembre de 2016, fue enviado al Coordinador General Jurídico de Gobierno del Estado, obteniéndose como respuesta vía Messenger el siguiente mensaje: "haremos lo necesario para incorporar estos elementos antes de entrar en operación el programa". Como resultado, uno de los motivos de anticonstitucionalidad de dicho programa, las foto-multas con responsabilidad solidaria del propietario del vehículo, al día de hoy no ha sido aplicado.

Logro 7. Para poner en contacto a los alumnos con una necesidad social y enseñarles que para incidir en su atención no siempre se requieren grandes aportaciones, se les invitó, a través del blog, a recolectar y entregar tapas plásticas a asociaciones civiles que proporcionan asistencia a niños con cáncer, instituciones que reciben recursos económicos que ayudan a sus fines, o a entregar las tapas a plantas recicladoras. La recolección y entrega se volvió permanente.

Esta experiencia hace conscientes a los autores de que herramienta y contenido significan sólo una luz muy tenue al final del túnel; que el uso de las TIC por sí mismo nada significa si primero no cambian los métodos de enseñanza del Derecho. El hecho que el alumno pase de ser recipiendario de la información a convertirse en participe activo de ella no será posible si primero los docentes no son capacitados en el uso de las TIc, en los nuevos métodos de enseñanza del Derecho y, sobre todo, en la comprensión de lo que significa que el propósito de la educación es insertar al alumno en una sociedad del conocimiento. 


\section{Conclusiones}

1. La experiencia demuestra que el uso de las tıc en la enseñanza del Derecho Penal es útil si se capacita primero al docente sobre sus alcances y fines.

2. Las TIC como herramientas permiten fortalecer el aprendizaje y potencializar el conocimiento adquirido en el aula.

3. Los docentes de la Unidad Académica de Derecho de la Universidad Autónoma de Zacatecas con el uso de las herramientas Tic didácticas fomentarán la comunicación y el aprendizaje en sus educandos de una manera sincrónica, telemática e interactiva.

4. El uso de las tıc permite la internacionalización de la Universidad, además de complementar el conocimiento del Derecho Penal, no sólo como una materia teórico-práctica, sino como una gran política criminal, entendida desde la implementación de la tecnología en la enseñanza, en la promulgación de leyes y en las políticas públicas a fin de garantizar la seguridad jurídica de la nación en el presente siglo.

\section{Referencias}

- ANUIES (2012). Inclusión con responsabilidad social. Una nueva generación de políticas de educación superior. México, Ciudad de México: ANuIES, Dirección de Medios Editoriales.

* Cázares Nieto, Enrique (Coord.) (2016). Pasos hacia una revolución en la enseñanza del derecho en el sistema romano-germánico. México, D.F.: UNAM-IIJ. Recuperado de: https://archivos.juridicas.unam.mx/www/bjv/libros/9/4297/26.pdf.

* García, I. Peña-López, l; Johnson, L., Smith, R., Levine, A. y Haywood, K. (2010). Informe Horizon: Edición Iberoamericana 2010. Austin, Texas: The New Media Consortium. Recuperado de: https://unesdoc.unesco.org/ark:/48223/pf0000141908.

* González Bello, Edgar Oswaldo y Castillo Navarro, Adolfo. La enseñanza universitaria en derecho con el uso didáctico de las Tıc. Díkê, Revista de Investigación en Derecho, Criminología y Consultoría Jurídica, 20, octubre 2016-marzo de 2017, 23-36. Recuperado de: http://www.apps.buap.mx/ojs3/index.php/dike/article/ view/235/212.

* Leyva Cordero, O., Ganga Contreras, F., Tejada Fernández y Hernández Paz, Abraham A. (Coords.) (2015). La formación de competencias en la educación superior, alcances y limitaciones desde referentes de México, España y Chile. s. I.: Tirant Humanidades. Recuperado de: http://eprints.uanl.mx/9784/1/Libro Formación por Competencias.pdf.

* Pese a crisis, UAz recibe certificación de competencia (12 de octubre de 2016). Revista Malinali. Recuperado de: http://revistamalinali.com/.

* UNESCO (2005). Hacia las sociedades del conocimiento. París, Francia: UNESCO. Recuperado de: http://openaccess.uoc.edu/webapps/o2/ bitstream/10609/2661/6/NMC HorizonReport IB 2010 def.pdf. 
* UNESCO (2016). Competencias y estándares TIC desde la dimensión pedagógica: Una perspectiva desde los niveles de apropiación de las Tic en la práctica educativa docente. Pontificia Universidad Javeriana-Cali. Recuperado de: http://www.unesco.org/ new/fileadmin/MULTIMEDIA/FIELD/Santiago/pdf/Competencias-estandares-TIC. pdf.

\section{Cómo citar este artículo}

* Gaytán Martínez, A., Pérez Pinto, J. A., Martínez Ponce, I. N. y Francisco Javier Itzamna Caamal Torre (2019). Las TIC en el estudio del derecho penal. Revista Digital Universitaria (RDU). Vol. 20, núm. 3 mayo-junio. Dol: http://doi.org/10.22201/ codeic.16076079e.2019.v20n3.a9.

Recepción: 23/08/17. Aprobación: 22/03/19 